The voluntary act of eye closure was a trigger for seizure activity, but visual input was an inhibitor. Unless visual input was prevented by allowing the eyes to close, seizures did not occur. Darkness alone was insufficient to elicit a seizure. The EEG seizure pattern consisted of generalized high-amplitude beta activity alternating with delta, and typical spike-and-wave activity was absent. (Barclay CL et al. Unusual form of seizures induced by eye closure. Epilepsia March/April 1993; 34: 289-293). (Reprints: Dr CL Barclay, Toronto Hospital, Western Division MP, 11th Fl, 399 Bathurst St, Toronto, Ontario, Canada M5T 2S8).

COMMENT. Seizures induced by eye closure are of two types: 1) those related to movement of the eyelids and occurring in photosensitive patients; and 2) a non-photosensitive type with scotosensitive epilepsy triggered by darkness alone or by obstruction of central visual fixation. The above patient required a combination of these two mechanisms for seizure induction.

\title{
NONEPILEPTIC SEIZURES DURING SLEEP
}

Nonepileptic (psychogenic or pseudo) seizures (NESs) were documented by video-electroencephalographic monitoring during apparent sleep in 12 patients reported from New York University School of Medicine. Onset was in childhood in 6 patients, and age at testing ranged from 11 to 67 years (mean, 33 yr). In addition to NESs unassociated with ictal EEG changes, 3 patients had EEG interictal epileptiform discharges and 1 had a complex partial seizure during monitoring, distinct from NES. Most NESs occurred within 2 hours of falling asleep or awakening. Provocative testing with suggestion reproduced nocturnal attacks in 9 patients. Seizures reported during sleep are not necessarily epileptic in nature. (Thacker $\mathrm{K}$ et al. Nonepileptic seizures during apparent sleep. Ann Neurol April 1993; $\underline{33:}$ 414-418). (Respond: Orrin Devinsky MD, Dept of Neurology, Hospital for Joint Diseases, 301 E 17th St, New York, NY 10003).

COMMENT. One-half of these patients had a history of childhood abuse. Four patients had evidence of epilepsy in addition to NESs.

The differential diagnosis in pseudoepileptic seizures is illustrated by case-reports from the Department of Neurology, University of Virginia Health Sciences Center, Charlottesville, VA.(Ozkara C, Dreifuss FE. Epilepsia March/April 1993; 34: 294-298). Epilepsy and pseudoepileptic seizures frequently coexist; the combination occurs in $20 \%$ before age 10 years and $60 \%$ after 10 years. Munchausen's syndrome often presents as pseudoepilepsy. Frontal lobe seizures have atypical patterns and may masquerade as pseudoseizures; these consist of thrashing, pedaling, kicking, or thrusting movements, an agitated appearance and utterance of loud expletives during sleep. 